\title{
Behavior Control of a New Designed Mobile Robot Based on Fuzzy Logic and Neuro Fuzzy Approaches for Monitoring Wall
}

\author{
Chokri Abdelmoula*, Hanen Rouabeh, Mohamed Masmoudi \\ EMC Research Group, National Engineering School of Sfax, Sfax, Tunisia \\ *Corresponding author's Email: chokri.abdelmoula@gmail.com
}

\begin{abstract}
This work describes the design and development of controllers based on artificial intelligence applied to a newly designed mobile robot type-vehicle to control behavior for monitoring wall. Two approaches have been developed and optimized to achieve this task. The first one is based on Fuzzy logic. This control algorithm combines different sensory information and provides a suitable control command allowing the mobile robot to follow the wall deviations. The second approach consists of the application of a hybrid-type Neuro-Fuzzy ANFIS controller for the same task. An important feature of this approach is that the controller combines the advantages of both Fuzzy logic and Neural Networks.The simulation results are presented and implemented with VHDL using ANFIS architecture.
\end{abstract}

Keywords: Mobile robot; Wall following; Fuzzy logic; Neural networks; Neuro-fuzzy; ANFIS

\section{Introduction}

In the last decade, the control of wheeled mobile robot motion has attracted an increasing interest among researchers. The evolution of electronic and computer systems has introduced a very important progress in autonomous mobile robots researches. Mobile robots are equipped with a set of sensors for perception and a set of actuators for motion and reaction. Navigation tasks of these robots can be controlled by the use of many command techniques. Among these, we note the advanced techniques based on intelligent algorithms. These algorithms based on artificial intelligence are used to solve problems of navigation, obstacle avoidance, intelligent parking and wall following behavior.

This paper discusses the optimization of a Fuzzy controller for the purpose of improving wall following behavior of a newly designed mobile robot. Firstly, the Fuzzy Logic controller was designed and simulated. Then, an optimized hybrid controller is developed using a Neuro-Fuzzy intelligent algorithm based on the Adaptative Neuro Fuzzy Inference System. This controller was designed and tested. In the final step, the obtained ANFIS architecture was implemented on FPGA using VHDL language.

In the literature many studies have focused on intelligent control of mobile robots. Researchers presented in $[1,2,3,4]$ have proposed various methods to control navigation tasks using different intelligent algorithms. Many extensions have been carried out on wall following behavior [5]. Farooq.U et al [6] have designed a fuzzy logic based intelligent controller for wall following behavior. They used three ultrasonic sensors mounted on left side of the robot. The fuzzy logic controller allows the robot to follow wall on its left side at a set distance. The inputs of the controller are the sensory data and outputs are motor command$s$ of the robot. Leehter Yaho and Yuan-Shiu Chen [7] have designed a type-2 fuzzy wall following controller for automated guided vehicle. The proposed controller is suitable for vehicles that use sonar system to measure distance to the wall. They stated that 
the type-2 fuzzy controller solved the noise problem presented by the sonar-based distance measurement. The parallel parking task has also attracted a great interest among researchers. Panomruttanarug.B et al [8] have presented a fuzzy logic based on autonomous parallel parking system. They used four infrared sensors for distances data acquisition. The distances are used as inputs to the fuzzy rules in order to calculate a proper steering angle while backing to achieve the parking process. The experimental results demonstrated the effectiveness of their approach. Zhi-Long Wang et al [9] proposed an autonomous parallel parking controller based on Neuro-Fuzzy approach for a car-like mobile robot. Their approach is based on the use of ultrasonic and compass sensors, mounted on the robot chassis, to decide on the turning angle. $\mathrm{K}$ Demirli and M Khoshnejad [10] have also developed a neuro-fuzzy model for autonomous parallel parking of a car like mobile robot. They have focused on the case when the parking space dimensions cannot be identified. The proposed approach uses the data from three sonar sensors mounted in the front left and right corner of the car to decide on the turning angle.

There are many other researches focusing on the obstacle avoidance task and navigation of mobile robots in crowded environment. Raguraman.S.M et al [11] have developed a fuzzy logic controller for mobile robot navigation in an indoor environment. The fuzzy system is designed with two basic behaviors- obstacle avoidance and a target seeking behavior. The inputs to the fuzzy logic controller are the desired direction of motion and the readings from the sensors whereas the outputs are the accelerations of robot wheels. Singh.M.K et al [12] used an adaptative Neuro-Fuzzy Inference System to control the navigation of a mobile robot in a dynamic environment. A learning algorithm based on neural network technique has been develope$\mathrm{d}$ to tune the parameters of fuzzy membership functions, which smooths the trajectory generated by the fuzzy logic system. Using the developed ANFIS controller, the mobile robots are able to avoid static and dynamic obstacles, and reach the target successfully in cluttered environments. Khaldoun K. Tahboub and Munaf S. N. Al-Din [13] have also developed a neurofuzzy reasoning scheme for mobile robot navigation. Their approach is based on decomposing a multidimensional fuzzy system into a set of simple parallel neural networks. The main advantage consists in reducing the number of if-then rules by introducing weighting factors to the sensor inputs. Four simple neural networks are used to determine these factors.
Each neural network is responsible for determining the weighting factor for one sensor input. Simulation results of this approach demonstrated the merits of the proposed system. Aleksandar Rodić et al [14] have presented a sensor-based intelligent mobile robot navigation control scheme. Their approach deals with combined, fuzzy and dynamic control of autonomous mobile robots and their motion in unknown environment with different obstacles. A detailed description of the proposed fuzzy inference system structure is presented and some implementation aspects are described as well as experimental control verification.

Many studies have been conducted on the hardware implementation of intelligent algorithms for mobile robot control. Chan Zhi and Muhammad Nasiruddin M. [15] have presented an obstacle avoidance program for mobile robot that incorporates a neuro-fuzzy algorithm using Altera Field Programmable Gate Array (FPGA) development board. A mobile robot serves as the test platform of the program. An ultrasonic sensor and servo motors are used as the test platform's sensing element and actuator, respectively. The neurofuzzy algorithm is successfully implemented on the Altera FPGA development board. A VHDL implementation of an intelligent navigation system using hybrid fuzzy controllers has been proposed by Nian Zhang et al in [16]. Two parallel fuzzy logic controllers are utilized to conquer the major mobile robot navigation challenges in narrow concave U-shaped obstacles, and deep narrow passage, and to track a target in an environment with varying obstacles. The result$s$ demonstrate the suitability of the FPGAs in control tasks for multiple fuzzy logic controllers. The implementation of the control units for the robot with FPGA is an important approach in the way for building a real prototype.

A solution to overcome limitations of fuzzy controller (non-existence of a standard method for extracting the Fuzzy rules) will be presented in this work. This problem can be solved by using the learning character of Neural Networks combined with Fuzzy inference system. To achieve this, we proceeded to develop an optimized hybrid controller using a Neuro-Fuzzy approach based on ANFIS architecture.

The remainder of this paper is organized as follows. Section 2 presents the kinematics modeling of the mobile robot. Section 3 illustrates the optimized controllers based on Fuzzy and Neuro Fuzzy mobile robot control. Section 4 gives explanatory details about the ANFIS hardware implementation. In Section 5, different results are explored and discussed. A summary 
of the key points concludes the paper in section 6.

\section{Kinematic Modeling of the mobile robot}

This work is developed for a vehicle -type mobile robot [17]. The rear wheels are fixed and the front ones are the steering wheels which are responsible for direction change. The movement of the robot is controlled by the steering angle $\phi$ of the front wheels. The kinematics modeling of the robot in the environment is shown in Fig. 1.

The frame $\mathrm{R}(\mathrm{O}, \mathrm{X}, \mathrm{Y})$ is related to space navigation. Point $\mathrm{M}\left(X_{M}, Y_{M}\right)$ is located at the middle of the two front wheels $\theta$ is the angle of orientation relative to the $\mathrm{X}$ axis.

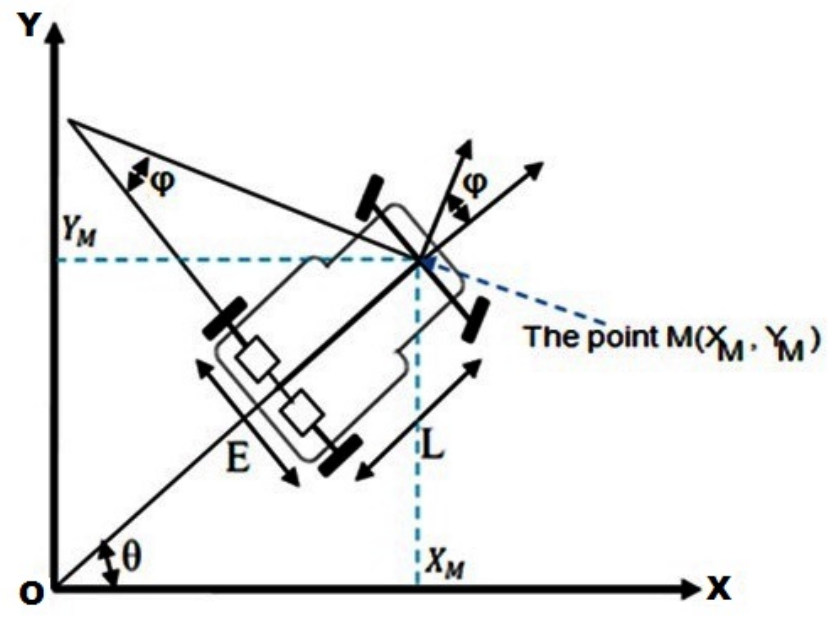

Figure 1 Kinematics modeling of the mobile robot

The motion of the robot in the $\mathrm{R}$ frame is described by:

$$
\left\{\begin{aligned}
d X_{M} & =v \cos (\theta) \\
d Y_{M} & =v \sin (\theta) \\
\theta & =\frac{v \sin (\phi)}{L}
\end{aligned}\right.
$$

Where the velocity $v$ is constant and equal $0.2 \mathrm{~m} / \mathrm{s}$. $\phi$ is the steering angle and $L$ the distance between front and rear wheels axis. By the discretization of these equations using the Euler method, the kinematics model becomes:

$$
\left\{\begin{aligned}
X_{M}(k+1) & =X_{M}(k)+h \cdot v \cdot \cos \theta(k) \\
Y_{M}(k+1) & =Y_{M}(k)+h \cdot v \cdot \sin \theta(k) \\
\theta(k+1) & =\theta(k)+h \cdot \frac{v \cdot \sin \phi(k)}{L}
\end{aligned}\right.
$$

Where $h$ is the sampling step.

\section{Fuzzy and Neuro-Fuzzy Robot Control}

\subsection{Development of the fuzzy controller}

The used Fuzzy controller for the mobile robot wall following behavior takes as inputs the two variables $E_{-} d$ and $E_{-}$theta shown in Fig. 2. In order to calculate these variables, we use two infrared sensors $\mathrm{Cd}_{1}$ and $\mathrm{Cd}_{2}$ installed on the right if the followed wal1 is on the right and vice verse. The different value $D d_{1}-D d_{2}$ and $d$ are used to calculate the orientation with the wall $E$ theta (where $D d_{1}$ and $D d_{2}$ present respectively the distance values returned by sensors $\mathrm{Cd}_{1}$ and $\mathrm{Cd}_{2}$, and $d$ the distance between the two sensors). The error on the desired distance $E_{-} d$ is also calculated using these values.

$$
\begin{aligned}
& E_{-} \text {thet } a=\tan g^{-1} \frac{\left(D d_{1}-D d_{2}\right)}{d} \\
& E_{-} d=D d_{1}-D_{-} \text {desired }
\end{aligned}
$$

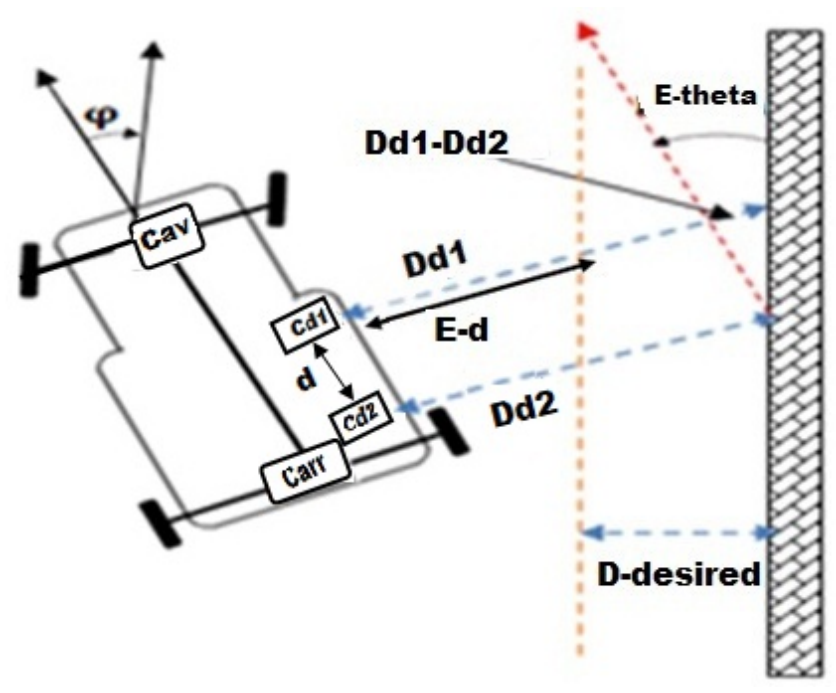

Figure 2 The input/output variables of the controller

The first step in designing the Fuzzy controller is the variables partition into Fuzzy sets. For the two input variables the number and type of membership functions are fixed as five triangular functions shown in Fig. 3 and Fig. 4. This distribution is based on the knowledge of the system and the desired accuracy.

The linguistic variables for both variables are noted: NB (Negative Big), NS (Negative Small), ZE (Zero), 
PS (Positive Small) and PB (Positive Big). The output $\phi$ is partitioned into five singletons. It is represented by five linguistic variables: NB (Negative Big), NS (Negative Small), ZE (Zero), PS (Positive Small), PB (Positive Big). Fig. 5 shows the partition of the output variable $\phi$.

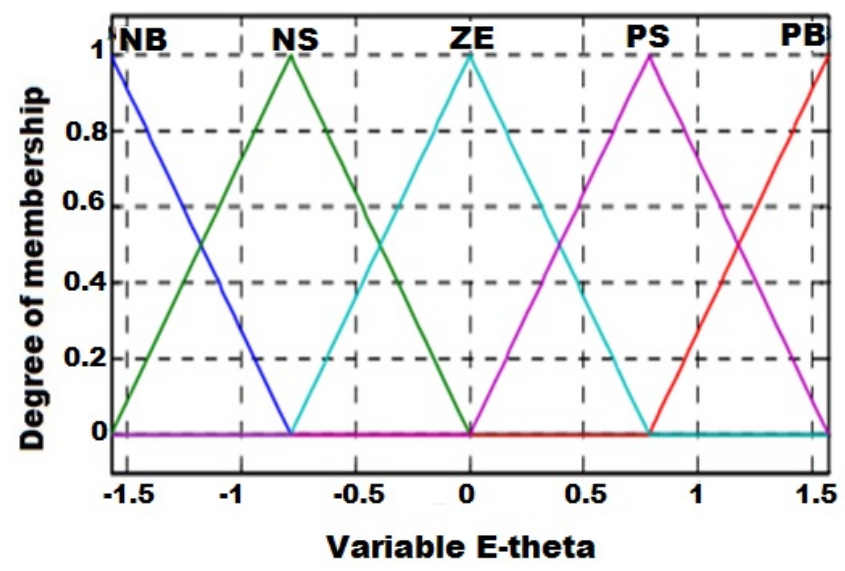

Figure 3 Fuzzy sets of the variable $E_{-}$thet $a(\mathrm{rad})$

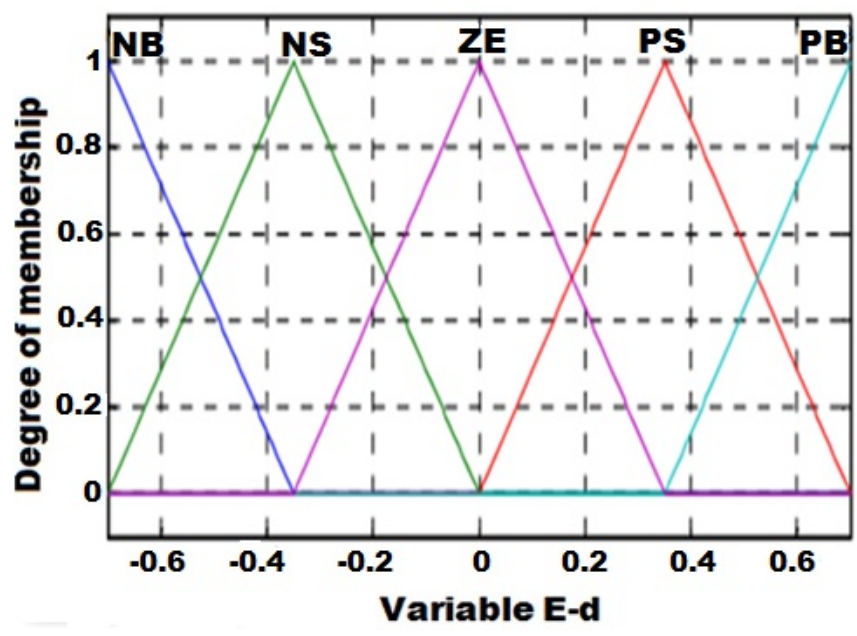

Figure 4 Fuzzy sets of the variable $E_{-} d(\mathrm{~m})$

Table 1 presents the Fuzzy rules that describe the behavior of the robot. There is no general method to extract these rules and their determination is based on both human experience and the conditions of the system to be controlled. We can distinguish different types of rules as follows:

$\operatorname{If}\left(E_{-} d\right.$ is NB $)$ and ( $E_{-}$theta is NS) then $(\phi$ is $\mathrm{PB})$

\subsection{Application of ANFIS for the wall following behavior}

The use of Fuzzy logic to control the mobile robot shows good results. However some weaknesses arise from the difficulty of obtaining knowledge from a human expert. To overcome this difficulty we decided to

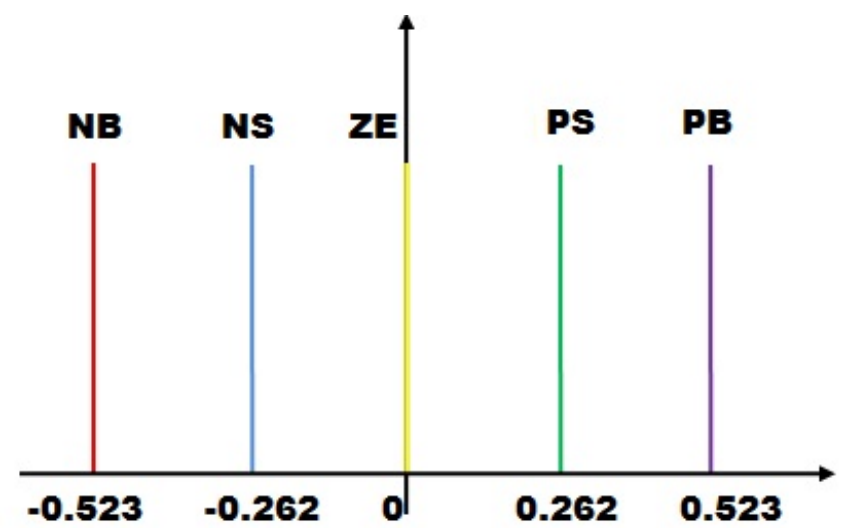

Figure 5 The output variable angle $\phi(\mathrm{rad})$

Table 1 Fuzzy rules

\begin{tabular}{|c|c|c|c|c|c|c|}
\hline & \multicolumn{5}{|c|}{$E_{-} d$} \\
\hline & & NB & NS & $\mathbf{Z E}$ & PS & PB \\
\hline \multirow{5}{*}{ 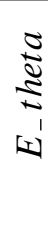 } & PB & $\mathrm{ZE}$ & NS & NS & NB & NB \\
\hline & PS & PS & $\mathrm{ZE}$ & NS & $\mathrm{NS}$ & $\mathrm{NB}$ \\
\hline & $\overline{\mathbf{Z E}}$ & PS & PS & $\overline{\mathrm{ZE}}$ & $\mathrm{NS}$ & $\mathrm{NS}$ \\
\hline & $\mathrm{NS}$ & $\overline{\mathrm{PB}}$ & PS & $\begin{array}{l}\mathrm{PS} \\
\end{array}$ & $\mathrm{ZE}$ & $\mathrm{NS}$ \\
\hline & $\mathrm{NB}$ & $\mathrm{PB}$ & $\mathrm{PB}$ & PS & PS & $\mathrm{ZE}$ \\
\hline
\end{tabular}

introduce the extracting knowledge concept without the need of expert. Automatic extraction of knowledge is based on the Neural Networks learning character. Thus appears the Neuro-Fuzzy approach that combines the advantages of both techniques.

In our work we have optimized a hybrid controller based on Neuro-Fuzzy approach called ANFIS (Adaptive Neuro Fuzzy Inference Based System). This architecture exploits the Sugeno Fuzzy model known as TSK (TSK Fuzzy model) proposed by Takagi, Sugeno and Kang [18] based on 5-layer neural network architecture. Each layer is designed to achieve a step in the Fuzzy inference system.

The concept of the wall following ANFIS controller is the same one used with the Fuzzy controller. The ANFIS network obtained is shown in Fig. 6.

This network has five layers:

The first layer: Consists of 6 neurons that classify each of the two input variables $E_{-} d$ and $E_{-}$thet $a$ into three Fuzzy sets, using «gbellmf» membership functions. Each neuron calculates its activation, which is equal to the degree of membership of the input element to a Fuzzy set.

The second layer: Consists of nine neurons, each neuron represents a Fuzzy rule and calculates its activation by a simple product. The outputs are $W_{i}$ with 
$i$ varying from 1 to 9 .

The third layer: Represents the normalization layer, consisting of 9 neurons, each one calculates the correspondent normalized weight. The outputs are $S_{i}$ with $i$ varying from 1 to 9 and given by the following equation:

$$
S_{i}=w_{i} / \sum_{i=1}^{9} w i
$$

The fourth layer: Consists of 9 neurons, used to calculate the consequences of the rules as follows:

$$
f_{i}=S_{i}\left(p_{i} E_{-} d+q_{i} E_{-} \text {theata }+r_{i}\right)
$$

With $p_{i}, q_{i}$ and $r_{i}$ represent the consequent parameters.

The fifth layer: This layer comprises a single neuron that computes the output of the ANFIS through the following sum:

$$
\text { Output }=\sum_{i=1}^{9} f i
$$

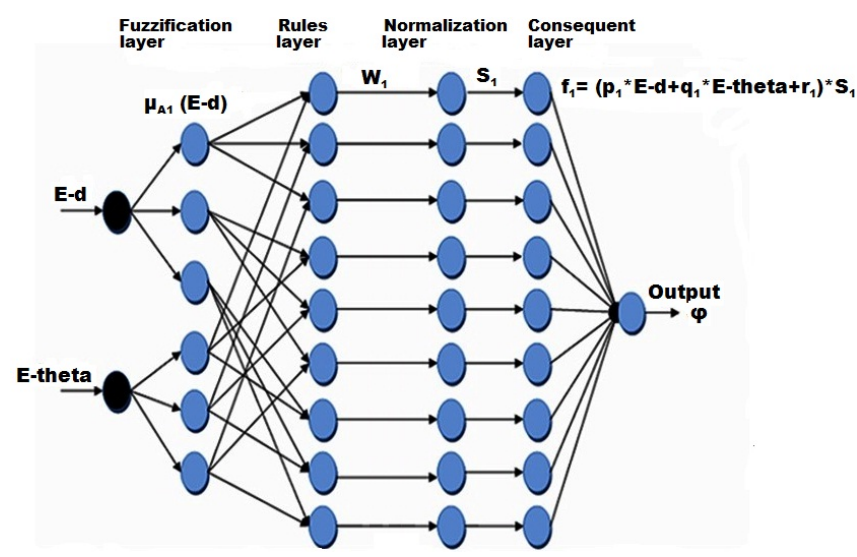

Figure 6 Architecture of the wall following ANFIS controller

The ANFIS training is achieved in two steps. The first stage is designed to adjust and calculate the consequent parameters using least square error algorith$\mathrm{m}$ by keeping the membership functions parameter$s$ fixed. The second one is designed to adjust and calculate the membership functions parameters with the back propagation algorithm by keeping the consequent parameters fixed [19].

The input variables are partitioned into three Fuzzy sets using the gbellmf function, which is described by the following equation:

$$
F(x, a, b, c)=\frac{1}{1+\left|\frac{x-c}{a}\right|^{2 b}}
$$

The distribution of these variables before and after ANFIS training for variable $E_{-} d$ is shown respectively in Fig. 7 and Fig. 8 Fig. 9 and Fig 10 show the distribution of $E_{-}$theta variable.

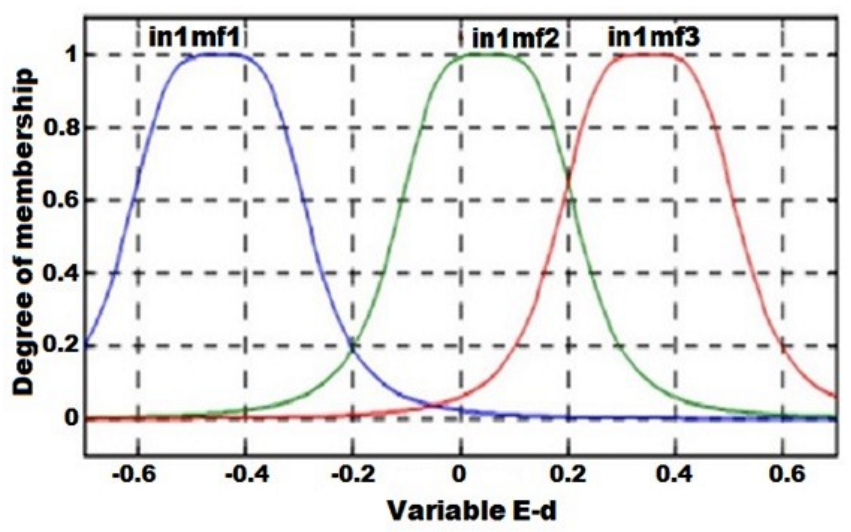

Figure 7 Variable $E_{-} d$ before learning

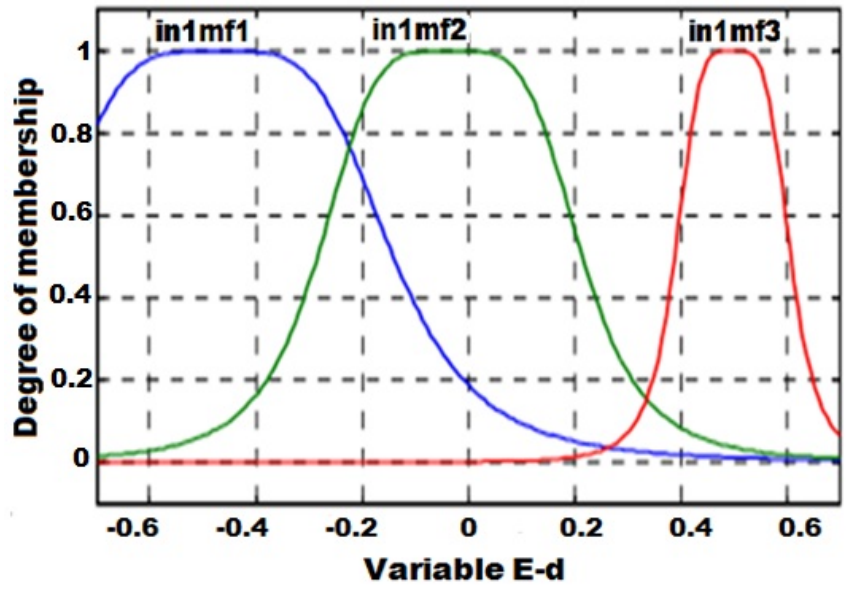

Figure 8 Variable $E_{-} d$ after learning

After optimizing the architecture using ANFIS controller, results are summarized and presented respectively in table 2 and table 3 . Table 2 shows the two variables $E_{-} d$ and $E_{-}$thet $a$ membership functions parameters before and after learning. Table 3 lists the rules consequent parameters obtained after the learning operation.

The ANFIS learning phase is done with a database of 1870 examples, the error obtained for this phase after 5000 iterations is about 0.0095964 . A database composed of 300 different examples is used to test the efficiency of the obtained architecture. The error obtained for testing phase is about 0.010132 , which is an acceptable error. 


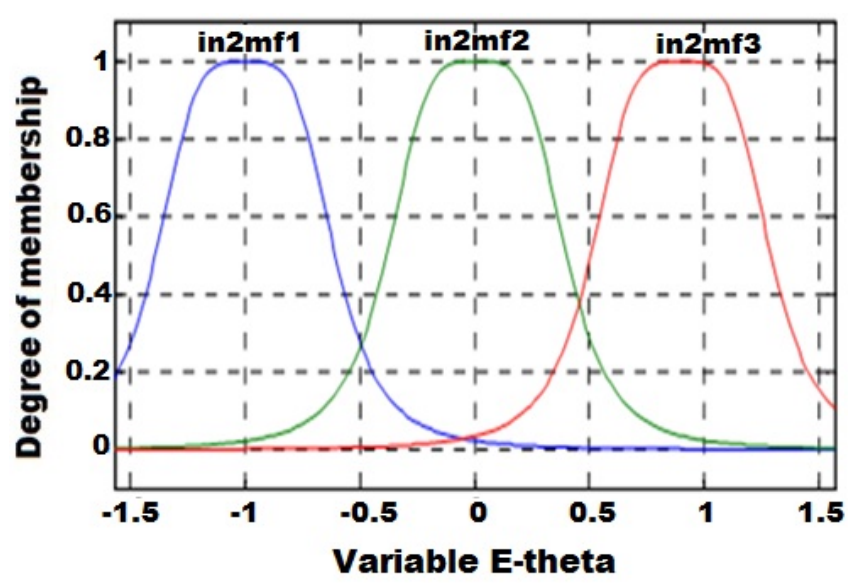

Figure 9 Variable $E_{-}$thet $a$ before learning

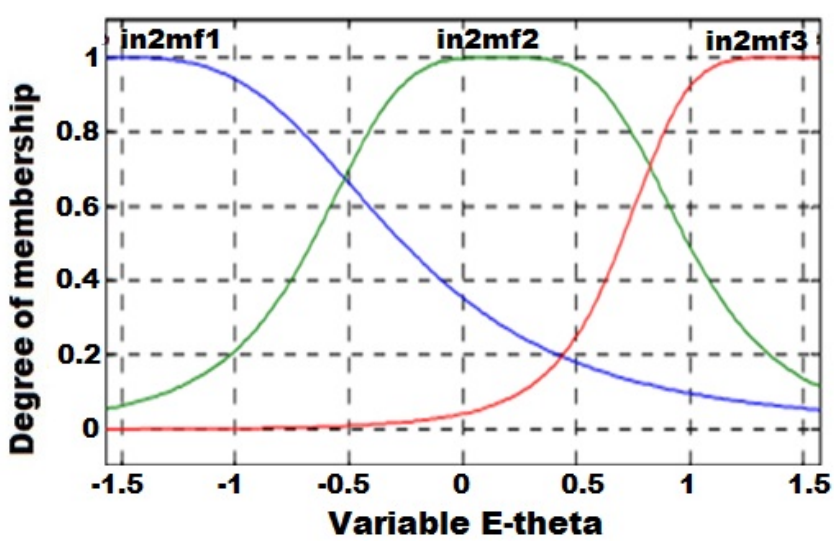

Figure 10 Variable $E_{-}$thet $a$ after learning

Table 2 Membership functions parameters before and after learning

\begin{tabular}{|c|c|c|c|c|c|c|}
\hline & \multicolumn{3}{|c|}{$\begin{array}{c}\text { Parameters } \\
\text { before learning }\end{array}$} & \multicolumn{3}{|c|}{$\begin{array}{l}\text { Parameters } \\
\text { after learning }\end{array}$} \\
\hline & $\mathbf{a}$ & b & c & $\mathbf{a}$ & b & c \\
\hline \multirow{3}{*}{$\begin{array}{l}0 \\
1 \\
1\end{array}$} & 0.175 & 2 & -0.45 & 0.3306 & 2.058 & -0.4716 \\
\hline & 0.175 & 2 & 0.05 & 0.2499 & 2.159 & -0.0344 \\
\hline & 0.175 & 2 & 0.35 & 0.1094 & 2.168 & 0.4988 \\
\hline \multirow{3}{*}{$\frac{\sqrt[a]{2}}{\sqrt[a]{2}}$} & 0.3927 & 2 & -0.983 & 1.288 & 1.628 & -1.549 \\
\hline & 0.3927 & 2 & 0 & 0.8291 & 1.921 & 0.1641 \\
\hline & 0.3927 & 2 & 0.883 & 0.7576 & 2.387 & 1.453 \\
\hline
\end{tabular}

Table 3 The consequent parameters obtained after learning

\begin{tabular}{|c|c|c|c|}
\cline { 2 - 4 } \multicolumn{1}{c|}{} & $\mathbf{p}$ & $\mathbf{q}$ & $\mathbf{r}$ \\
\hline$S_{1}$ & 8.658 & 3.431 & 3.698 \\
\hline$S_{2}$ & -4.975 & -1.94 & -1.309 \\
\hline$S_{3}$ & -1.097 & -0.4317 & 0.03616 \\
\hline$S_{4}$ & -2.219 & -0.6785 & -0.322 \\
\hline$S_{5}$ & 0.3801 & -1.524 & -0.1554 \\
\hline$S_{6}$ & -6.489 & -5.768 & 5.892 \\
\hline$S_{7}$ & -9.963 & 2.529 & 7.701 \\
\hline$S_{8}$ & 5.054 & -0.6862 & -4.072 \\
\hline$S_{9}$ & -24.91 & 0.9169 & 19.04 \\
\hline
\end{tabular}

\section{Hardware Implementation of the ANFIS}

After setting all parameters of the ANFIS architecture, we implement it on FPGA with VHDL language using the Altera Quartus II environment. In order to obtain an optimized architecture, we choose the data encoding type which ensures the necessary accuracy. The natural numbers between 0 and $2^{n-1}$ coded in natural binary with n-bit, and negative integers encoded in two's complement. For real numbers we have chosen floating point encoding. We used the floating point representation normalized by the standard IEEE754 single precision with 32-bits.

Five blocks are created to implement the ANFIS.

Block 1: Fuzzification block, it has six ROM type memories that contain the discretization of the six membership functions.

Block2: Rules layer's block, it makes a simple product between the two inputs to give the output weights $W_{i}$.

Block3: Normalization block takes as input the weight$\mathrm{s} W_{i}$ and gives as output the normalized weights, described by equation (5).

Block4: Presents the fourth network layer, it is responsible for calculating the rules consequences by equation (6). All calculations are performed by the IEEE 754 floating point representation.

Block5: Calculates the output $\phi$ by the sum of $f_{i}$ as represented by equation (7).

The complete block diagram of the ANFIS network used is shown in Fig. 11.

For the implementation we have used a cyclone III EP3C120F7807 FPGA. The compilation summary of the presented ANFIS VHDL prototype is described in Table 4. 


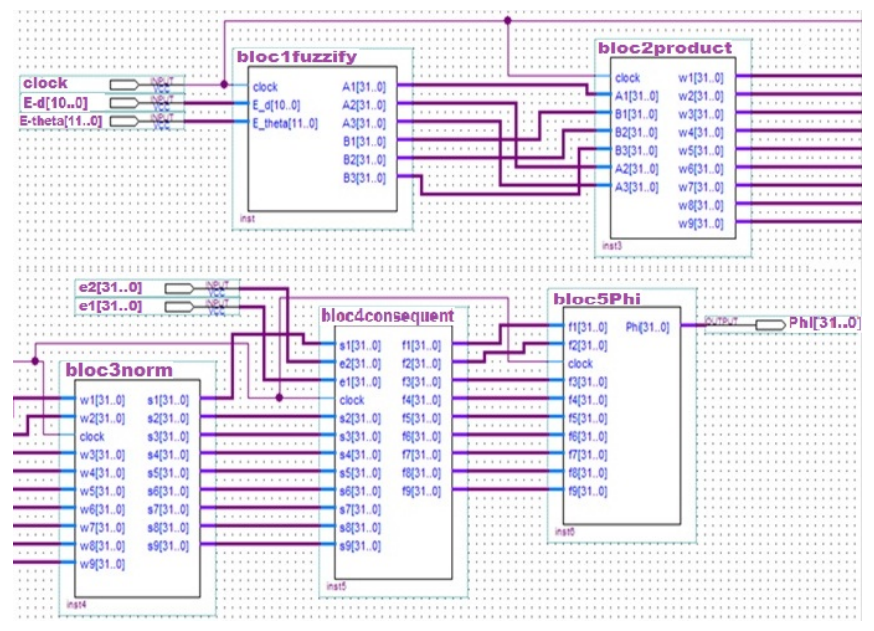

Figure 11 Complete architecture of the ANFIS network

Table 4 The ANFIS hardware resources

\begin{tabular}{|c|c|}
\hline Total memory bits & $601.859 / 3.981 .312(15 \%)$ \\
\hline Total pins & $120 / 532(23 \%)$ \\
\hline Total logic elements & $80.893 / 119.088(68 \%)$ \\
\hline
\end{tabular}

\section{Simulation Results}

This section presents the MATLAB simulation results for both controllers. The Fuzzy controller was tested as an example where the followed wall doesn't contain any deviation. The robot direction is parallel to the wall but the distance between them is greater than the desired distance. The ANFIS controller was tested for a more complicated form of wall that contains many deviations.

\subsection{Simulation results of fuzzy controller}

Fig. 12 and Fig. 13 show respectively, the trajectory and the output steering angle generated by the Fuzzy controller for an example where the initial parameters have the following values: $\mathrm{X}=0, \mathrm{Y}=0$ and angle $\theta=$ $\pi / 2$.

The robot's initial position is characterized by the angle $\theta=\pi / 2$ ( $\theta$ angle of orientation relative to the axis $(\mathrm{O}, \mathrm{X}))$ and a zero angle with the axis $(\mathrm{O}, \mathrm{Y})$, so robot's direction is parallel to axis $(\mathrm{O}, \mathrm{Y})$. In addition to that, the followed wall is parallel to this axis, so we can say that the robot's direction is parallel to the wall but the distance between them is greater than the desired distance $D_{-}$desired $=0.4 \mathrm{~m}$. As a consequence the robot will move forward by turning right to reach the desired distance. When approaching this distance it begins to rotate in opposite direction, so the angle $\phi$ will increase in the negative direction when the robot approaches the desired distance. This angle will re-

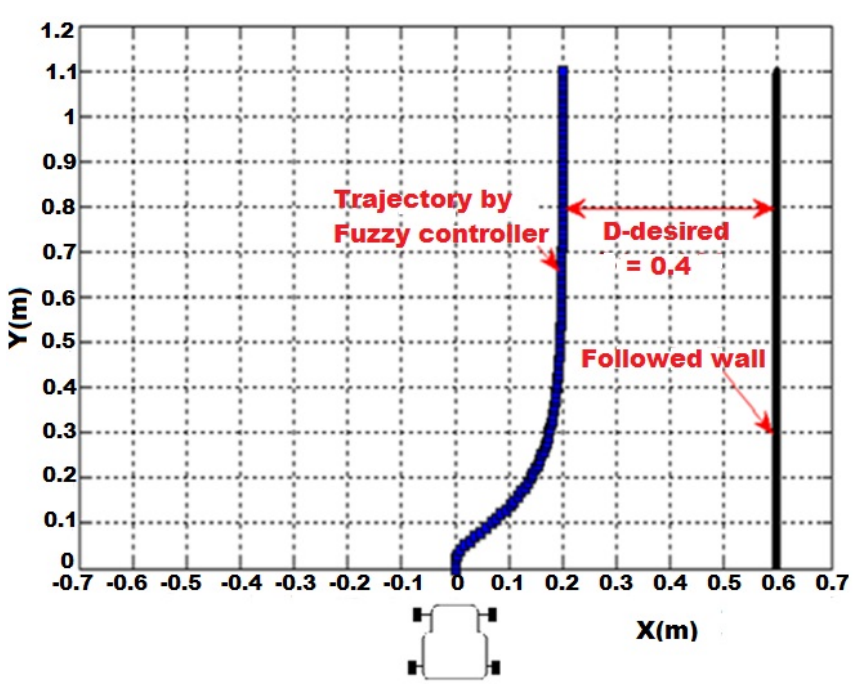

Figure 12 Robot trajectory by the fuzzy controller

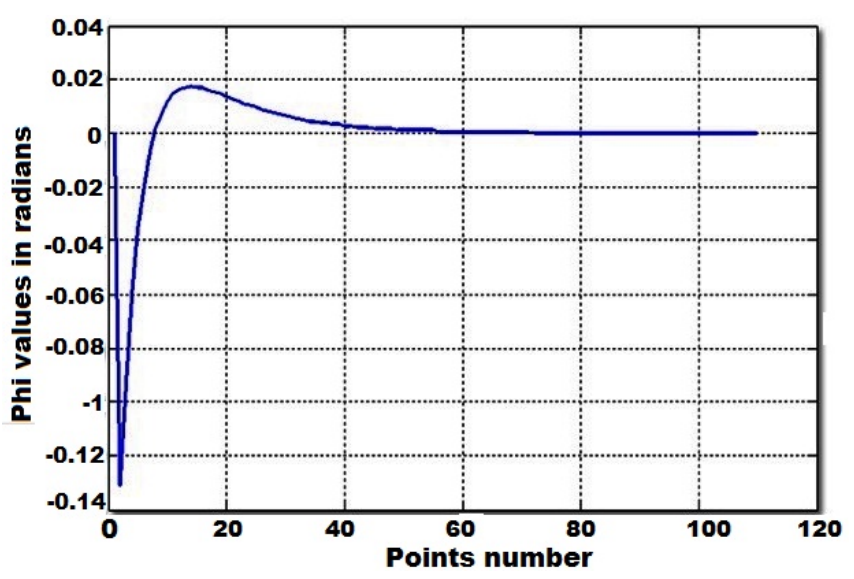

Figure 13 Variation of the angle $\phi(\mathrm{rad})$

turn gradually to zero until it keeps a direction parallel to the wall where the angle is zero for parallel follow with the desired fixed distance which is $0.4 \mathrm{~m}$.

\subsection{Simulation results of ANFIS}

After training the operation of the ANFIS network and setting the parameters of membership function$s$ and consequent parameters, we tested the network with a new test database containing 300 examples. The parameters of the initial robot's position are: $X=$ $0.2 \mathrm{~m}, \mathrm{Y}=0 \mathrm{~m}$ and angle $\theta=\pi / 2$, the wall to be followed is at a distance of $0.6 \mathrm{~m}$ from the robot. This distance is greater than the desired distance which is $0.3 \mathrm{~m}$. The wall has 3 deviations, so the robot must follow these deviations keeping the desired distance fixed. The results are obtained adequately to the desired movement in the environment of the mobile robot and are presented respectively in Fig. 14 and Fig. 15. 


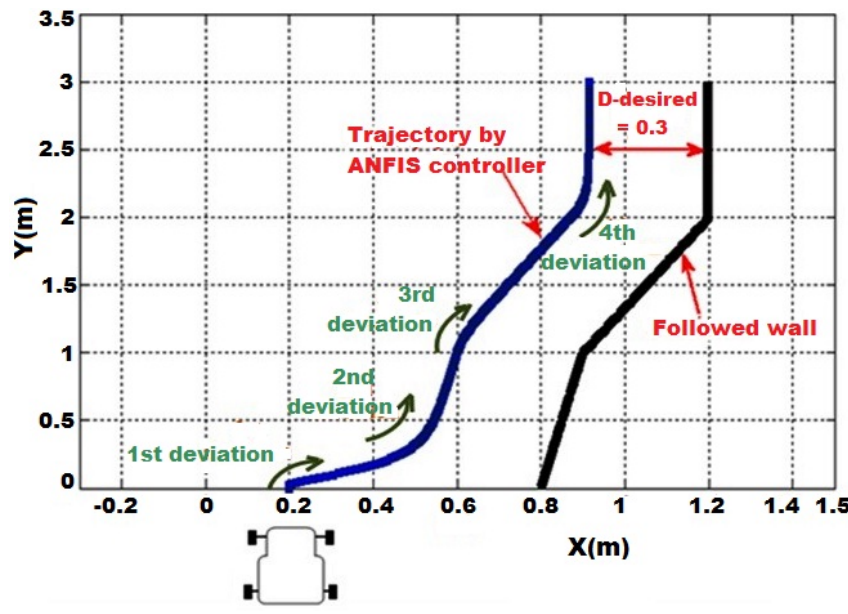

Figure 14 Robot trajectory by the ANFIS controller

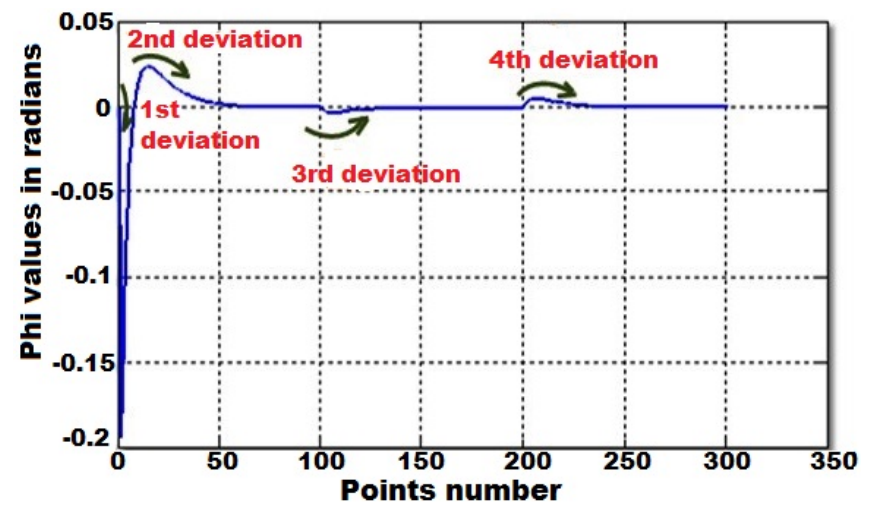

Figure 15 Variation of angle $\phi(\mathrm{rad})$

\subsection{The ANFIS testing and validation with VHDL}

To check the validity of the ANFIS architecture implemented with VHDL language, a functional simulation is carried out. We compared the theoretical values given by the MATLAB program with those given by VHDL. We take the following values as an example of the input values to test the ANFIS controller in MATLAB:

$E_{-} d=0.121$ et $E_{-}$thet $a=0.225$. The value of the corresponding output generated by the controller in MATLAB is $\phi=-0.262$.

Then we validate this result by simulating the implemented architecture of ANFIS with the same inputs. The simulation results are presented in Fig. 16.

The value of $\phi$ obtained by the VHDL simulation is: $\phi=10111110100001011011001001111110$

This value converted to real number with single precision is: $\phi=-0.2611274$.

The two values of $\phi$ obtained by MATLAB and VHDL are very close. Table 5 presents some other values tested and compared with the theoretical values:

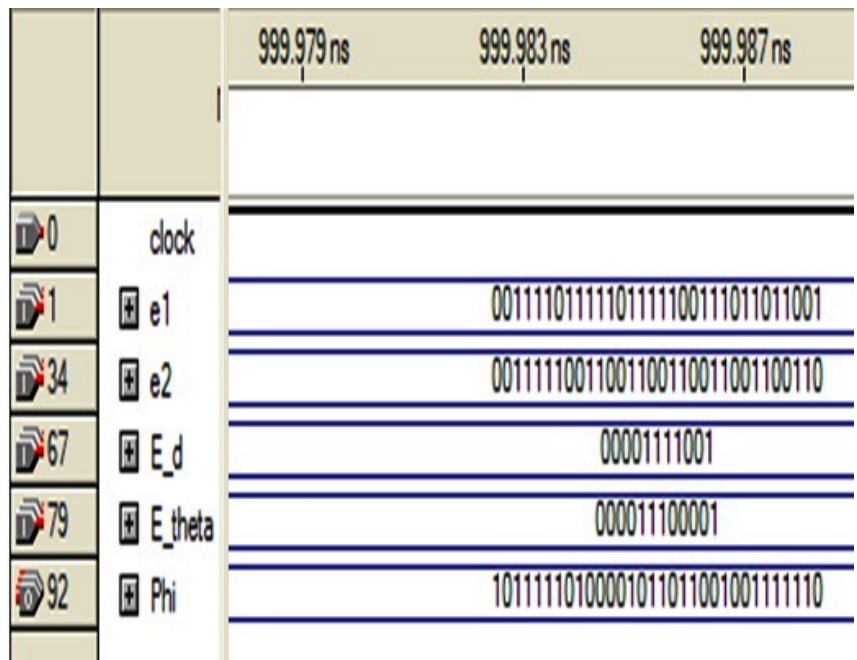

Figure 16 Simulation results of ANFIS controller

Table 5 Examples of test

\begin{tabular}{|c|c|c|}
\cline { 2 - 3 } \multicolumn{1}{c|}{} & $\begin{array}{c}\text { Theoritical } \\
\phi\end{array}$ & $\begin{array}{c}\text { VHDL } \\
\text { values }\end{array}$ \\
\hline$E_{-} d=0.38$ & -0.141 & -0.1413377 \\
$E_{-}$thet $=-0.12$ & & 1011111000010000101110 \\
& & 1011010100 \\
\hline$E_{-} d=-0.122$ & -0.112 & -0.11199709 \\
$E_{-}$thet $a=0.23$ & & 1011110111100101010111 \\
& & 1010111011 \\
\hline$E_{-} d=0.753$ & -0.213 & -0.21320324 \\
$E_{-}$thet $a=0.17$ & & 1011111001011010010100 \\
& & 0111110011 \\
\hline$E_{-} d=-0.35$ & 0.48 & 0.4803706 \\
$E_{-}$thet $a=-0.175$ & & 0011111011110101111100 \\
& & 1100100011 \\
\hline
\end{tabular}

\subsection{Discussion}

The optimized Fuzzy controller shows good result$\mathrm{s}$ for different forms of wall as presented previously. Fuzzy logic shows ability to formalize and simulate the operator's expertise for robot control. It allows taking into account the different variables of the problem. However, it is limited by some disadvantages such as the non-existence of a standard and a systematic method to transform the experiences of an expert to a base rule, with no general procedure for choosing the optimal number of rules. The optimized controller requires much time to set the suitable number and the more efficient type of membership function$\mathrm{s}$ and Fuzzy rules for wall following behavior control. The use of the hybrid-type ANFIS control system for monitoring wall allows the automatic extrac- 
tion of knowledge without the need of human expert. The ANFIS combines the features of Fuzzy logic with that of Neural Networks. The learning character of these networks has to adjust the membership functions parameters and rules consequences parameters to get an optimized architecture. The simulation of the resulting ANFIS controller shows efficiency and performance in the robot control. Compared with the Fuzzy controller, the ANFIS gives effective results with 3 Fuzzy sets for each of the two inputs and 9 Fuzzy rules, instead of 5 Fuzzy sets and 25 rules for the Fuzzy controller. The character of learning can lead to an optimal architecture more easily and without wasting much time for the formalization of Fuzzy rules.

\section{Conclusion}

This work describes the optimization and the development of Fuzzy logic and Neuro-Fuzzy based controllers for an operation of monitoring wall applied to a newly designed mobile robot. The Fuzzy controller allows the robot prototype to follow the wall deviations, keeping a desired fixed distance with respect to it. The simulation results of this controller are satisfactory, but it is not possible to avoid long time to set the architecture that best meets the problem. As a solution, a Neuro-Fuzzy control system was applied to the same task. A hybrid-type ANFIS controller is designed and tested. The test results show more efficiency and performance when using this type of control. The ANFIS architecture obtained was implemented using VHDL on FPGA. The implementation on FPGA has been chosen based on its low cost, reliability and reconfigurable characters. The use of these two controllers shows efficiency in laboratory application; through the major advantages they possess particular Neuro-Fuzzy approach that combines the advantages of both techniques.

This application is a part of our work in developing autonomous mobile robot navigation systems. Our perspectives are oriented towards developing a multisensory based architecture for on road intelligent vehicles navigation.

\section{References}

[1] B.K. Shim, J.H. Kim, I.M. Park and S.H. Han,“An intelligent control of non-holonomic mobile robot based on fuzzy perception", In: International Conf. On Control Automation and Systems (ICCAS), pp.2111-2114, 2010.
[2] S. Jie and Z. Jinqiang, "The Research of Intelligent Control System of Autonomous Mobile Robot", In: EEEE '09 International Conference, pp.241-244, 2009.

[3] J.G. Kang, S.Y. An, S. Kim and Se.Y. Oh,"Sonar based Simultaneous Localization and Mapping using a Neuro Evolutionary Optimization”, In: International Joint Conference IJCNN, pp. 1516-1523, June 2009.

[4] M. Gunes and A. Baba,"Speed and position control of autonomous mobile robot on variable trajectory depending on its curvature", Journal of Scientific and Industrial Research, Vol.68, pp. 513-521, 2009.

[5] D. Floreano, J.Codjevaq, A.Martinoli, F.Mondada, and J.D.Nicoud,"Design Control and Applications of Autonomous Mobile Robot”, In: International Series on Microprocessor-Based and Intelligent Systems Engineering, Vol.18, pp.159-186, 1999.

[6] M.K. Singh, D.R. Parhi and J.K. Pothal,"ANFIS Approach for Navigation of Mobile Robots", In:ARTCom '09, International Conference on Advances in Recent Technologies in Communication and Computingpp. 727-731, 2009.

[7] L.Yaho and Y.S Chen,"A type-2 fuzzy controller for automatic guided vehicle wall-following control”, In: International Conference ICIC 2010, Vol.1, No.1, pp.77-83, 2010.

[8] B.Panomruttanarug, S.Tungporntawee, P.Thongsuk and K.Higuchi, "An Emulation of Autonomous ParallelParking System using Fuzzy Logic Control", In: International Joint Conference ICCAS-SICE, pp.4548-4552, 2009.

[9] Z.L. Wang, C.H. Yang and T.Y. Guo, “The Design of an Autonomous Parallel Parking Neuro-Fuzzy Controller for a Car Like Mobile Robot", In: International Conference SICE , pp. 2593-2599,2010.

[10] K.Demirli and M.Khoshnejad,"Autonomous parallel parking of a car-like mobile robot by a neuro-fuzzy sensor-based controller", Journal of Fuzzy Sets and Systems, Vol.160, pp.2876-2891, 2009.

[11] S.M. Raguraman, D. Tamilselvi, and N. Shivakumar,"Mobile robot navigation using Fuzzy logic controller", In: International Conf. On Control, Automation, Communication and Energy Conservation, pp.1-5, 2009.

[12] U. Farooq, A. Khalid, M. Amar, A. Habiba, S. Shafique and R. Noor,"Design and low cost implementation of a fuzzy logic controller for wall following behavior of a mobile robot", In: International Conf. On Signal Processing Systems (ICSPS),Vol.2, pp.740-746, 2010.

[13] K.A. Tahboub and M. S. N. Al-Din,"A Neuro-Fuzzy Reasoning System for Mobile Robot Navigation", Jordan Journal of Mechanical and Industrial Engineering, Vol.3, No.1, pp.77-88, 2009. 
[14] A. Rodic, K. Addi and M. Jezdimirovic, "SensorBased Intelligent Navigation and Control of Autonomous Mobile Robots for Advanced Terrain Missions", In: Scientific Technical Review, Vol.60, No.2, pp.7-15, 2010.

[15] C.Z. Wei and M.N. Mahyuddin,"Neuro-fuzzy algorithm for obstacle avoidance mission of a mobile robot using FPGA", In: International Conf. On Innovative Technologies in Intelligent Systems and Industrial Applications, pp.305-310, 2009.

[16] N. Zhang, R. Kamdem, E. Ososanya, W. Mahmoud, and W. Liu,"VHDL implementation of the hybrid fuzzy logic controllers with FPGA", In: International Conf. On Intelligent Control and Information Processing (ICICIP), pp.5-10, 2010.

[17] C. Abdelmoula, F. Chaari and M. Masmoudi,"A new design of a robot prototype for intelligent navigation and parallel parking", Journal of Automation, Mobile Robotics and Intelligent Systems, Vol.3, No.2, pp.4758, 2009.

[18] F. Rastegar, B.N. Araabi, and C. Lucas, "An Evolutionary Fuzzy Modeling Approach for ANFIS Architecture ", In: IEEE Congress on Evolutionary Computation, Vol.3, pp.2182-2189, 2005.

[19] http://www.2shared.com/file/xEXr11dD/CHAPITRE III_RNA_ ANFIS.html 